\title{
A NOTE ON SPACES WHICH HAVE COUNTABLE TIGHTNESS
}

\author{
Woo Chorl Hong
}

\begin{abstract}
In this paper, we introduce closure operators $[\cdot]_{c}$ and $[\cdot]_{a}$ on a space and study some relations among $[\cdot]_{c},[\cdot]_{a}$ and countable tightness. We introduce the concepts of a strongly sequentially closed set and a strongly sequentially open set and show that a space $X$ has countable tightness if and only if every strongly sequentially closed set is closed if and only if every strongly sequentially open set is open. Finally we find a generalization of the weak Fréchet-Urysohn property which is equivalent to countable tightness.
\end{abstract}

\section{Introduction}

All spaces under considered here are always assume to be infinite and $T_{1}$. Our terminology is standard and follows [3] and [12].

Let $X$ be a topological space. For each subset $A$ of $X$, we use $\bar{A}$ for the closure of $A$ in $X$. Let $\mathbb{N}$ denote the set of all natural numbers and $\left(x_{n}\right)$ a sequence of points of a set.

We recall that a space $X$ has countable tightness $[1,2,6,7,10]$ (also called closure countable [12] or c-space [9]) if and only if for each subset $A$ of $X$ and each $x \in \bar{A}$, there exists a countable subset $C$ of $A$ such that $x \in \bar{C}$. A space $X$ is sequential $[1,2,4,7,10]$ if and only if for each subset $A$ of $X$ which is not closed in $X$, there are $x \in \bar{A} \backslash A$ and a sequence $\left(x_{n}\right)$ of points of $A$ such that $\left(x_{n}\right)$ converges to $x$ in $X$.

Obviously, a countable space as well as a space in which every subspace is separable has countable tightness. It is clear that every first countable space is sequential and every sequential space has countable tightness. But, the converses need not be true in general (see $[4,7,10]$ ).

The function $[\cdot]_{\text {seq }}$ from the power set $\mathfrak{P}(X)$ of $X$ into $\mathfrak{P}(X)$ itself defined by for each subset $A$ of $X,[A]_{\text {seq }}=\left\{x \in X:\left(x_{n}\right)\right.$ converges to $x$ in $X$ for some sequence $\left(x_{n}\right)$ of points of $\left.A\right\}$ is called the sequential closure operator on

Received January 11, 2010; Revised November 26, 2010.

2010 Mathematics Subject Classification. 54A20, 54B15, 54C10, 54D55, 54D99.

Key words and phrases. countable tightness, c(a)-closure operators, strongly sequentially closed, strongly sequentially open, and weak Fréchet-Urysohn property.

This paper was supported for two years by Pusan National University Research Grant. 
$X$ [2]. A space $X$ is Fréchet-Urysohn [1, 2, 7, 10] (also called Fréchet [4, 5] or closure sequential [12]) if and only if for each subset $A$ of $X$ and each $x \in \bar{A}$, there exists a sequence $\left(x_{n}\right)$ of points of $A$ such that $\left(x_{n}\right)$ converges to $x$ in $X$.

It is well-known that the sequential closure operator $[\cdot]_{\text {seq }}$ satisfies the Kuratowski topological closure axioms except for idempotency in general, for each subset $A$ of a space $X, A \subset[A]_{\text {seq }} \subset \bar{A}$, and $X$ is Fréchet-Urysohn (sequential) if and only if for each subset $A$ of $X,[A]_{\text {seq }}=\bar{A}$ (resp. for each subset $A$ of $X$ which is not closed in $\left.X,[A]_{s e q} \backslash A \neq \emptyset\right)$. See $[2,7]$.

S. P. Franklin $[4,5]$ studied properties of sequential spaces and FréchetUrysohn spaces, introduced a sequentially open set and a sequentially closed set and showed that a space $X$ is sequential if and only if every sequentially open set in $X$ is open if and only if every sequentially closed set in $X$ is closed. F. Siwiec [10] noted several properties of spaces which have countable tightness. V. I. Malykhin and G. Tironi [8] introduced the weak Fréchet-Urysohn property and showed the weak Fréchet-Urysohn property is strictly stronger than countable tightness. Recently, the author [7] showed some relations among generalized metric spaces and a space which has countable tightness.

In this paper, we study some properties of spaces which have countable tightness. We introduce closure operators $[\cdot]_{c}$ and $[\cdot]_{a}$ on a space and show that $[\cdot]_{c}$ is a topological closure operator on $X,[\cdot]_{c}=[\cdot]_{a}$ and a space $X$ has countable tightness if and only if for each subset $A$ of $X,[A]_{c}=\bar{A}$ if and only if for each subset $A$ of $X$ which is not closed in $X,[A]_{c} \backslash A \neq \emptyset$. We also show that for a space $(X, \mathfrak{T})$ with a topology $\mathfrak{T}$, we have the following:

(1) $\left(X, \mathfrak{T}_{[\cdot]_{c}}\right)$ has countable tightness, where $\mathfrak{T}_{[\cdot]_{c}}$ is the topology for $X$ induced by $[\cdot]_{c}$.

(2) If $(X, \mathfrak{T})$ does not have countable tightness, then $\mathfrak{T} \varsubsetneqq \mathfrak{T}_{[\cdot]_{c}}$ and $\mathfrak{T}_{[\cdot]_{c}}=\mathfrak{D}$, where $\mathfrak{D}$ is the discrete topology for $X$ if and only if every countable subset of $X$ is closed in $(X, \mathfrak{T})$.

(3) $\mathfrak{T}_{[\cdot]_{c}}$ is the smallest countable tight expansion of $\mathfrak{T}$; that is, if a space $\left(X, \mathfrak{T}^{*}\right)$ has countable tightness and $\mathfrak{T} \subset \mathfrak{T}^{*}$, then $\mathfrak{T}_{[\cdot]_{c}} \subset \mathfrak{T}^{*}$.

We introduce the concepts of strongly sequential closedness and strongly sequential openness which are stronger than sequential closedness and sequential openness, respectively and show that $X$ has countable tightness if and only if every strongly sequentially closed set in $X$ is closed if and only if every strongly sequentially open set in $X$ is open.

Finally we find a generalization of the weak Fréchet-Urysohn property which is equivalent to countable tightness.

\section{Results}

We begin by introducing closure operators $[\cdot]_{a}$ and $[\cdot]_{c}$ on a space $X$. Recall that a sequence $\left(x_{n}\right)$ of points of a space $X$ accumulates to a point $x$ in $X$ [3] if and only if for each open set $U$ in $X$ containing $x$ and each $m \in \mathbb{N}$, there 
exists $n \in \mathbb{N}$ such that $m \leqq n$ and $x_{n} \in U$; that is, for each open set $U$ in $X$ containing $x,\left(x_{n}\right)$ is frequently in $U$.

Definition 2.1. (1) The function $[\cdot]_{a}: \mathfrak{P}(X) \rightarrow \mathfrak{P}(X)$ defined by for each subset $A$ of a space $X,[A]_{a}=\left\{x \in X:\left(x_{n}\right)\right.$ accumulates to $x$ in $X$ for some sequence $\left(x_{n}\right)$ of points of $\left.A\right\}$ is called the a-closure operator on $X$.

(2) The function $[\cdot]_{c}: \mathfrak{P}(X) \rightarrow \mathfrak{P}(X)$ defined by for each subset $A$ of a space $X,[A]_{c}=\{x \in X: x \in \bar{C}$ for some countable subset $C$ of $A\}$ is called the c-closure operator on $X$.

We have directly that for each subset $A$ of a space $X, A \subset[A]_{\text {seq }} \subset[A]_{a} \subset$ $[A]_{c} \subset \bar{A}$ and for each countable subset $C$ of $X,[C]_{c}=\bar{C}$.

Lemma 2.2. Let $X$ be a space. Then we have

(1) $[\cdot]_{c}$ is a topological closure operator on $X$.

(2) $[\cdot]_{c}=[\cdot]_{a}$.

Proof. (1) It is obvious that $[\cdot]_{c}$ satifies the Kuratowski topological closure axioms except for idempotency and for each subset $A$ of $X,[A]_{c} \subset\left[[A]_{c}\right]_{c}$. Hence it suffices to show that for each subset $A$ of $X,\left[[A]_{c}\right]_{c} \subset[A]_{c}$. Suppose that there exists a subset $A$ of $X$ such that $\left[[A]_{c}\right]_{c} \nsubseteq[A]_{c}$ and let $x \in\left[[A]_{c}\right]_{c} \backslash$ $[A]_{c}$. Then since $x \in\left[[A]_{c}\right]_{c}$, there exists a countable subset $C_{x}$ of $[A]_{c}$ such that $x \in \bar{C}_{x}$. Either $C_{x} \backslash A$ is finite or infinite. If $C_{x} \backslash A$ is finite, then obviously $x \in[A]_{c}$, which is a contradiction. If $C_{x} \backslash A$ is infinite, then $C_{x} \cap\left([A]_{c} \backslash A\right)$ is countably infinite. Let $C_{x} \cap\left([A]_{c} \backslash A\right)=\left\{x_{n}: n \in \mathbb{N}\right\}$. Then for each $n \in \mathbb{N}$, since $x_{n} \in[A]_{c}$, there is a countable subset $D_{n}$ of $A$ such that $x_{n} \in \overline{D_{n}}$. Thus, we have

$$
\begin{aligned}
\overline{C_{x}} & =\overline{C_{x} \cap A} \cup \overline{C_{x} \cap\left([A]_{c} \backslash A\right)} \subset \overline{C_{x} \cap A} \cup \overline{\left(\cup\left\{\overline{D_{n}}: n \in \mathbb{N}\right\}\right)} \\
& \subset \overline{C_{x} \cap A} \cup \overline{\overline{\left(\cup\left\{D_{n}: n \in \mathbb{N}\right\}\right)}}=\overline{C_{x} \cap A} \cup \overline{\left(\cup\left\{D_{n}: n \in \mathbb{N}\right\}\right)} \\
& \subset \overline{\left(C_{x} \cap A\right) \cup\left(\cup\left\{D_{n}: n \in \mathbb{N}\right\}\right)} .
\end{aligned}
$$

Note that $\left(C_{x} \cap A\right) \cup\left(\cup\left\{D_{n}: n \in \mathbb{N}\right\}\right)$ is a countable subset of $A$ and $x \in \overline{\left(C_{x} \cap A\right) \cup\left(\cup\left\{D_{n}: n \in \mathbb{N}\right\}\right)}$. It follows that $x \in[A]_{c}$, which is also a contradiction.

(2) Obviously, for each subset $A$ of $X,[A]_{a} \subset[A]_{c}$.

Conversely, let $x \in[A]_{c}$. Then, by the definition of $[\cdot]_{c}$, there exists a countable subset $C$ of $A$ such that $x \in \bar{C}$. Since $X$ is $T_{1}, C \cap U$ is infinite for each open set $U$ in $X$ containing $x$. Hence, let $C=\left\{x_{n}: n \in \mathbb{N}\right\}$, considered as a sequence, the sequence $\left(x_{n}\right)$ accumulates to $x$ in $X$. Thus we have $x \in[A]_{a}$.

Theorem 2.3. Let $X$ be a space. Then the following statements are equivalent:

(1) X has countable tightness.

(2) For each subset $A$ of $X,[A]_{c}=\bar{A}$.

(3) For each subset $A$ of $X$ which is not closed in $X,[A]_{c} \backslash A \neq \emptyset$. 
Proof. $(1) \Leftrightarrow(2)$ It is obvious.

$(2) \Rightarrow(3)$ Let $A$ be a non-closed subset of $X$. Then $\bar{A} \backslash A \neq \emptyset$ and hence, by condition (2), $[A]_{c} \backslash A \neq \emptyset$.

$(3) \Rightarrow(2)$ Suppose it is not. Then there exists a space $X$ which does not have countable tightness and satisfies condition (3). Hence there exists a subset $A$ of $X$ such that $\bar{A} \backslash[A]_{c} \neq \emptyset$. Clearly, $\overline{[A]_{c}}=\bar{A}$ and so $[A]_{c}$ is not closed. By condition $(3),\left[[A]_{c}\right]_{c} \backslash[A]_{c} \neq \emptyset$. This contradicts Lemma 2.2(1).

From Theorem 2.3 and Lemma 2.2(2), we have immediately the following corollary and hence omit the proof.

Corollary 2.4. Let $X$ be a space. Then the following statements are equivalent:

(1) $X$ has countable tightness.

(2) For each subset $A$ of $X,[A]_{a}=\bar{A}$.

(3) For each subset $A$ of $X$ which is not closed in $X,[A]_{a} \backslash A \neq \emptyset$.

Theorem 2.5. Let $(X, \mathfrak{T})$ be a space with a topology $\mathfrak{T}$. Then we have

(1) $\left(X, \mathfrak{T}_{[\cdot]_{c}}\right)$ has countable tightness, where $\mathfrak{T}_{[\cdot]_{c}}$ is the topology for $X$ induced by $[\cdot]_{c}$.

(2) If $(X, \mathfrak{T})$ does not have countable tightness, then $\mathfrak{T} \varsubsetneqq \mathfrak{T}_{[\cdot]_{c}}$ and $\mathfrak{T}_{[\cdot]_{c}}=\mathfrak{D}$, where $\mathfrak{D}$ is the discrete topology for $X$ if and only if every countable subset of $X$ is closed in $(X, \mathfrak{T})$.

(3) If $(X, \mathfrak{T})$ does not have countable tightness, then $\mathfrak{T}_{[\cdot]_{c}}$ is the smallest countable tight expansion of $\mathfrak{T}$.

Proof. (1) It follows from Lemma 2.2(1) and Theorem 2.3.

(2) It is obvious that if $(X, \mathfrak{T})$ does not have countable tightness, then $\mathfrak{T} \varsubsetneqq$ $\mathfrak{T}_{[\cdot]_{c}} \cdot$

Let $C$ be a countable subset of $(X, \mathfrak{T})$. Then clearly $[C]_{c}=\bar{C}$ and hence, by hypothesis; $\mathfrak{T}_{[\cdot]_{c}}=\mathfrak{D}, \bar{C}=C$. Thus $C$ is closed in $(X, \mathfrak{T})$.

Conversely, suppose that there exists a space $(X, \mathfrak{T})$ such that every countable subset of $X$ is closed in $(X, \mathfrak{T})$ and $\mathfrak{T}_{[\cdot]_{c}} \neq \mathfrak{D}$. Then there exists $x \in X$ such that $\{x\} \notin \mathfrak{T}_{[\cdot]_{c}}$. Since $X \backslash\{x\}$ is not closed in $\left(X, \mathfrak{T}_{[\cdot]_{c}}\right),[X \backslash\{x\}]_{c}=X$ and hence there exists a countable subset $C_{x}$ of $X \backslash\{x\}$ such that $x \in \overline{C_{x}}$. By hypothesis, $\overline{C_{x}}=C_{x}$. Thus we have $x \in C_{x} \subset(X \backslash\{x\})$, which is a contradiction.

(3) Suppose that there are a space $(X, \mathfrak{T})$ which does not have countable tightness and a topology $\mathfrak{T}^{*}$ for $X$ such that $\mathfrak{T} \subset \mathfrak{T}^{*} \varsubsetneqq \mathfrak{T}_{[\cdot]_{c}}$ and $\left(X, \mathfrak{T}^{*}\right)$ has countable tightness. Then clearly there exists a subset $A$ of $X$ such that $[A]_{c} \varsubsetneqq$ $\bar{A}^{*} \subset \bar{A}$, where $\bar{A}^{*}$ is the closure of $A$ in the space $\left(X, \mathfrak{T}^{*}\right)$. Let $x \in \bar{A}^{*} \backslash[A]_{c}$. Then since $\left(X, \mathfrak{T}^{*}\right)$ has countable tightness and $x \in \bar{A}^{*}$, there exists a countable subset $C$ of $A$ such that $x \in \bar{C}^{*}$. Since $\mathfrak{T} \subset \mathfrak{T}^{*}, \bar{C}^{*} \subset \bar{C}$ and so $x \in \bar{C}$. Thus $x \in[A]_{c}$, which is a contradiction.

Recall that a subset $A$ of a space $X$ is sequentially closed [4] if and only if no sequence of points of $A$ converges to a point not in $A$. A subset $U$ of a space $X$ 
is sequentially open [4] if and only if each sequence of points of $X$ converging to a point in $U$ is eventually in $U$. It is well-known that a space $X$ is sequential if and only if every sequentially closed set in $X$ is closed if and only if every sequentially open set in $X$ is open (see $[4,10]$ ).

Definition 2.6. Let $X$ be a space and $A \subset X$.

(1) $A$ is a strongly sequentially closed set in $X$ if and only if no sequence of points of $A$ accumulates to a point not in $A$.

(2) $A$ is a strongly sequentially open set in $X$ if and only if each sequence of points of $X$ accumulating to a point in $A$ is frequently in $A$.

It is obvious that every closed (open) set is strongly sequentially closed (strongly sequentially open) and every strongly sequentially closed (strongly sequentially open) set is sequentially closed (resp. sequentially open). But, by the following examples we know that the converses need not be true in general.

Note that $A$ is a sequentially closed (strongly sequentially closed) set in $X$ if and only if $A=[A]_{\text {seq }}$ (resp. $A=[A]_{c}$ ).

Example 2.7. Let $X=\left[0, \omega_{1}\right]$ be the space of ordinals with the ordinary order topology, where $\omega_{1}$ is the first uncountable ordinal. Then $X$ is a Hausdorff compact space, but does not have countable tightness [7, 11]. Clearly, $X \backslash\left\{\omega_{1}\right\}$ is not closed. Note that $\bar{C} \subset X \backslash\left\{\omega_{1}\right\}$ for each countable subset $C$ of $X \backslash\left\{\omega_{1}\right\}$. Hence $\left[X \backslash\left\{\omega_{1}\right\}\right]_{c}=X \backslash\left\{\omega_{1}\right\}$ and so $\left[X \backslash\left\{\omega_{1}\right\}\right]_{\text {seq }}=\left[X \backslash\left\{\omega_{1}\right\}\right]_{c}=X \backslash\left\{\omega_{1}\right\}$. Thus $X \backslash\left\{\omega_{1}\right\}$ is sequentially closed as well as strongly sequentially closed.

Example 2.8. Let $X=\{(0,0)\} \cup(\mathbb{N} \times \mathbb{N})$. We define a topology $\mathfrak{T}$ for $X$ by for each $(m, n) \in X \backslash\{(0,0)\},\{(m, n)\} \in \mathfrak{T}$ and $(0,0) \in U \in \mathfrak{T}$ if and only if for all but a finite number of integers $m$, the sets $\{n \in \mathbb{N}:(m, n) \notin U\}$ are each finite. Hence each point $(m, n) \in X \backslash\{(0,0)\}$ is isolated and each open neighborhood of $(0,0)$ contains all but a finite number of points in each of all but a finite number of columns (see Arens-Fort space in [11]). Then $X$ is a Hausdorff space which has countable tightness and there is a unique nonisolated point $(0,0)$ in $X$, but not sequential $[7,11]$. Note that there does not exist any sequence of points of $\mathbb{N} \times \mathbb{N}$ converging to $(0,0)$. Hence it is clear that $\{(0,0)\}$ is sequentially open in $X$, but not strongly sequentially open. Also it is clear that $\mathbb{N} \times \mathbb{N}$ is sequentially closed in $X$. On the other hand, since $(0,0) \in \overline{\mathbb{N} \times \mathbb{N}}=X$, by Lemma $2.2(2)$, we have that there exists a sequence $\left(x_{n}\right)$ of points of $\mathbb{N} \times \mathbb{N}$ such that $\left(x_{n}\right)$ accumulates to $(0,0) \notin \mathbb{N} \times \mathbb{N}$. Thus $\mathbb{N} \times \mathbb{N}$ is not strongly sequentially closed in $X$.

Example 2.9. Let $X=\{z\} \cup \mathbb{R}$, where $\mathbb{R}$ is the set of all real numbers and $z \notin \mathbb{R}$. We define a topology $\mathfrak{T}$ for $X$ by for each $x \in \mathbb{R},\{x\} \in \mathfrak{T}$ and $z \in U \in \mathfrak{T}$ if and only if $\mathbb{R} \backslash U$ is countable. Then $X$ is Hausdorff and $z$ is a unique nonisolated point in $X$ [7]. Clearly, $\{z\}$ is sequentially open in $X$ as well as strongly sequentially open in $X$, but not open.

Lemma 2.10. Let $X$ be a space and $A \subset X$. Then we have 
(1) $[A]_{c}$ is a strongly sequentially closed set in $X$.

(2) $A$ is strongly sequentially closed in $X$ if and only if $X \backslash A$ is strongly sequentially open in $X$.

Proof. (1) By Lemma 2.2(1), $\left[[A]_{c}\right]_{c}=[A]_{c}$ and hence it holds.

(2) Suppose that there exists a strongly sequentially closed subset $A$ of $X$ such that $X \backslash A$ is not strongly sequentially open in $X$. Then since $A$ is a strongly sequentially closed set in $X,[A]_{c}=A$ and hence $X \backslash[A]_{c}$ is not a strongly sequentially open set in $X$. We have that there exists a sequence $\left(x_{n}\right)$ of points of $X$ such that $\left(x_{n}\right)$ accumulates to a point $x \in X \backslash[A]_{c}$ and $\left(x_{n}\right)$ is not frequently in $X \backslash[A]_{c}$. It follows that there is a sequence $\left(y_{n}\right)$ of points of $[A]_{c}$ which accumulate to $x$. Hence, $x \in\left[[A]_{c}\right]_{a}$. By Lemma 2.2(1) and (2), $\left[[A]_{c}\right]_{a}=\left[[A]_{c}\right]_{c}=[A]_{c}$ and so $x \in[A]_{c}$. This is a contradiction.

Conversely, suppose that there exists a subset $A$ of $X$ such that it is strongly sequentially open in $X$ and $X \backslash A$ is not strongly sequentially closed. Then clearly $X \backslash A \varsubsetneqq[X \backslash A]_{c}$ and hence there exist $x \in A$ and a sequence $\left(x_{n}\right)$ of points of $X \backslash A$ such that $\left(x_{n}\right)$ accumulates to $x$ in $X$. Since $A$ is strongly sequentially open in $X,\left(x_{n}\right)$ is frequently in $A$. This is a contradiction.

Theorem 2.11. Let $X$ be a space. Then the following statements are equivalent:

(1) X has countable tightness.

(2) Every strongly sequentially closed set in $X$ is closed.

(3) Every strongly sequentially open set in $X$ is open.

Proof. $(1) \Rightarrow(2)$ Suppose that there exist a space $X$ of countable tightness and a strongly sequentially closed subset $A$ of $X$ with $\bar{A} \backslash A \neq \emptyset$. Let $x \in \bar{A} \backslash A$. Then, by Corollary 2.4, there exists a sequence $\left(x_{n}\right)$ of points of $A$ such that $\left(x_{n}\right)$ accumulates to $x$. It follows that $A$ is not strongly sequentially closed, which is a contradiction.

$(2) \Rightarrow(3)$ It follows directly from Lemma $2.10(2)$.

$(3) \Rightarrow(1)$ Suppose that there exists a space $X$ which satisfies condition (3) and does not have countable tightness. Then by Theorem 2.3 there exists a subset $A$ of $X$ such that $\bar{A} \backslash[A]_{c} \neq \emptyset$. By Lemma 2.10(1) and (2), $X \backslash[A]_{c}$ is strongly sequentially open in $X$. Thus by condition $(3)[A]_{c}$ is closed in $X$ and so $\bar{A}=[A]_{c}$, which is a contradiction.

Recall that a space $X$ is weakly Fréchet-Urysohn [8] if and only if for a subset $A$ of $X$ and $x \in X$, if $x \in \bar{A} \backslash A$, then there exists a countable infinite disjoint family $\mathfrak{F}$ of finite subsets of $A$ such that for each neighborhood $V$ of $x$, the subfamily $\{F \in \mathfrak{F}: F \cap V=\emptyset\}$ is finite. In [8], V. I. Malykhin and G. Tironi showed that the following implications hold: sequentiality $\Rightarrow$ weak Fréchet-Urysohn property $\Rightarrow$ countable tightness.

Finally we find a generalization of the weak Fréchet-Urysohn property which is equivalent to countable tightness. 
Theorem 2.12. A space $X$ has countable tightness if and only if for a subset $A$ of $X$ and $x \in X$, if $x \in \bar{A} \backslash A$, then there exists a countable infinite disjoint family $\mathfrak{F}$ of finite subsets of $A$ such that for each neighborhood $V$ of $x$, the subfamily $\{F \in \mathfrak{F}: F \cap V \neq \emptyset\}$ is infinite.

Proof. Let $x \in \bar{A} \backslash A$. Then since $X$ is $T_{1}$ and has countable tightness, we have that there exists a sequence $\left(x_{n}\right)$ of distinct points of $A$ such that $\left(x_{n}\right)$ accumulates to $x$. Now let $F_{n}=\left\{x_{n}\right\}$ for all $n \in \mathbb{N}$. Then, $\mathfrak{F}:=\left\{F_{n}: n \in\right.$ $\mathbb{N}\}$ is a countable infinite disjoint family of finite subsets of $A$ and for each neighborhood $V$ of $x,\left\{n \in \mathbb{N}: F_{n} \cap V \neq \emptyset\right\}$ is infinite since $\left(x_{n}\right)$ accumulates to $x$. Thus it holds.

Conversely, let $x \in \bar{A}$. By Theorem 2.3(2), it suffices to show that $x \in[A]_{c}$. If $x \in A$, then obviously $x \in \overline{\{x\}}$ and so $x \in[A]_{c}$. Hence, it remains to prove the case where $x \in \bar{A} \backslash A$. In this case, by hypothesis, there exists a countable infinite disjoint family $\mathfrak{F}=\left\{F_{n}: n \in \mathbb{N}\right\}$ of finite subsets of $A$ such that for each neighborhood $V$ of $x,\left\{n \in \mathbb{N}: F_{n} \cap V \neq \emptyset\right\}$ is infinite. It follows that $\cup_{n \in \mathbb{N}} F_{n}$ is a countable subset of $A$ and $\left(\cup_{n \in \mathbb{N}} F_{n}\right) \cap V$ is infinite. Thus, $x \in[A]_{c}$ and therefore, $X$ has countable tightness.

From the definition of weak Fréchet-Urysohn property and Theorem 2.12, we obtain directly the following corollary and hence omit the proof.

Corollary 2.13 ([8, Proposition 1.4]). Every weakly Fréchet-Urysohn space has countable tightness.

Remark 2.14. (1) It is well-known that the two properties: Fréchet-Urysohn property and sequentiality are completely determined by $[\cdot]_{s e q}$. From Theorem 2.3 and Corollary 2.4, we have that $[\cdot]_{c}$ ( or $[\cdot]_{a}$ ) determines completely countable tightness. Hence, a question naturally arises in connection with $[\cdot]_{\text {seq }}$ and $[\cdot]_{c}$ $\left(\right.$ or $\left.[\cdot]_{a}\right)$ : Is there any reasonable generalization of countable tightness which is completely determined by $[\cdot]_{c}\left(\right.$ or $\left.[\cdot]_{a}\right)$ ?

(2) From the fact $[\cdot]_{\text {seq }}$ does not satisfy idempotence [2, p. 13, Example 13], we know directly that for sequentially closed sets and sequentially open sets, Lemma 2.10(2) need not be true in general.

(3) A space $X$ is accumulation complete [10] if and only if each sequence $\left(x_{n}\right)$ of points of $X$ accumulating to a point $x$ of $X$ has a subsequence which converges to $x$. It is well-known that a space $X$ being accumulation complete is equivalent to every countable subspace of $X$ being Fréchet-Urysohn. Thus we know easily that every accumulation complete space which has countable tightness is Fréchet-Urysohn (and hence sequential).

Acknowledgement. The author wish to thank the referee for his/her very kind detailed comments in improving the exposition of the paper.

\section{References}

[1] A. V. Arhangel'skii, Topological Function Spaces, Mathematics and its Applications (Soviet Series), 78. Kluwer Academic Publishers Group, Dordrecht, 1992. 
[2] A. V. Arhangel'skii and L. S. Pontryagin (Eds.), General Topology I, Encyclopaedia of Mathematical Sciences, 17. Springer-Verlag, Berlin, 1990.

[3] J. Dugundji, Topology, Allyn and Bacon, Inc., Boston, 1970.

[4] S. P. Franklin, Spaces in which sequences suffice, Fund. Math. 57 (1965), 107-115.

[5] _ Spaces in which sequences suffice II, Fund. Math. 61 (1967), 51-56.

[6] H. Z. Hdeib, On spaces which have countable tightness, Questions Answers Gen. Topology 6 (1988), no. 1, 11-20.

[7] W. C. Hong, Generalized Fréchet-Urysohn spaces, J. Korean Math. Soc. 44 (2007), no. $2,261-273$.

[8] V. I. Malykhin and G. Tironi, Weakly Fréchet-Urysohn and Pytkeev spaces, Topology Appl. 104 (2000), no. 1-3, 181-190.

[9] T. W. Rishel, A class of spaces determined by sequences with their cluster points, Portugal. Math. 31 (1972), 187-192.

[10] F. Siwiec, Generalizations of the first axiom of countability, Rocky Mountain J. Math. 5 (1975), 1-60.

[11] L. A. Steen and J. A. Seebach, Jr., Counterexamples in Topology, Springer-Verlag, Berlin, 1978.

[12] A. Wilansky, Topology for Analysis, Ginn and Company, 1970.

Department of Mathematics Education

Pusan National University

PUSAN 609-735, Korea

E-mail address: wchong@pusan.ac.kr 